\title{
KNOWLEDGE BASED QUESTION ANSWERING
}

\author{
Michael J. Pazzani and Carl Engelman \\ The MITRE Corporation \\ Bedford, MA 01730
}

ABSTRACT

The natural language database query syotem incorporated in the RNOBS interactive plenning eyotem comprises a dictionary driven parser, APE-II, and script interpreter which yield a conceptual dependency conceptualization as a representation of the maning of user input. A conceptualization pattern antehing production syotem then deterwines and executes a procedure for extracting the desired information from the database. In contrast to syatex driven Q-A oyotews, e.g., those based on ATI parsers, APE-II is driven bottom-up by expectations aseciated vith word mennings. The procesaing of a query is based on the contents of several knowledge sources including the dictionary entries (partial conceptualization: and their expectations), frames representing conceptual dependency primitives, script. which contain stereotypical knowledge about planning takk used to infer states enabling or resulting frow actions, and two production syotem rule bases for the infereace of implicit case fillers, and for determining the responsive dacabase search. The goals of this approach, all of which are currently at least partially achieved, include utilizing oimilar representations for questions with similar meanings but widely varying gurface seructures, developing a powerful mechanism for the disambiguation of words with multiple meaninge and the determination of pronoun referents, anowering questions which require inferences to be understood, and interpreting ellipsea and ungrammetical utterances.

\section{THE SETTING}

The KNOBS [Engelman, 1980] demonstration system is an experimental expert system providing consultant services to an Air Force tactical air mission planner. The KNOBS database consists of several nets of frames, implemented within an excension of FRL [Roberts, 1977], representing both individual and generic classes of targets, resources, and planned missions. The KNOBS system supports a planner by checking the consistency of plan components, enumerating or ranking possible choices for plan components, or automatically generating a complete plan. Because these activities are accomplished by means of rules and constraints expressible in English, KNOBS will hopefully be a relatively easy system to learn.
For the same reasons, it is also being considered a an aid to train aiesion planners. The natural language subsyaten of RHOBS plays several roles including those of database query, database update, command language, plan definition, and the addition or modification of production system rules representing domain knowledge. The nost developed of these is database query, upon which this paper vill focus.

The balance of this paper will first outline the use of conceptual dependency and mention some prior related work and then describe the several knowledge sources and the parts they play in the paraing of the input query. Finally, it will describe the mathod of deriving the appropriate database search and output response as well as a script-based approach to interpretting commands.

\section{USE OF CONCEPTUAL DEPENDENCY}

APE-II utilizes Conceptual Dependency theory [Schank, 1972] to represent the meaning of questions. Once the meaning of a question has been found, the quection is answered by a rule based syotem whose tests are CD patterns and whose actions execute dacabase queries.

We feel it is important to represent the meaning in this manner for several reasons. First, the canonical weaning representation enables questions which have different surface expressions, but the same meaning, to be answered by the same mechanibm. This is not only of theoretical significance, but is also a practical matter as is requires less effort to produce a robust system.

Because people do not always say precisely what they mean, inferences may be required to explicate misaing information. This inference process can also utilize the canonical meaning representation. Finally, finding the referent of a nominal which is modified by a relative clause is, in some cases, oimilar to question answering although the syntactic constructions used differ. As a resule of this similarity, the question answering productions can also be used for determining the referents of a relative clauge. The conversation with KNOBS (whose database is fictional) in Fig. I illustrates these points.

The first question is represented in the same manner as "Does Ramstein have $F-4 G^{\prime} s$ ?" and would be answered by the same rule. The second question, 
USER: Are there $F-46^{\circ}$, at Rame tein?

RNOBS: RAMSTEIN has F-4Ga.

USER: Can its fighters reach the target?

RNOBS: $8-15$ s can reach BE 50301 from RAMSTEIN

F-4G. and F-4C. Can not reach BESO301 from RAMSTEIN.

USER: Which SCL which are carried by an F-4C contain ECM? KNOBS: B1, 87 and B8.

Figure 1. A Question Answering Interchange within XNOBS.

after resolving the pronominal reference, requires an inference to find the location from which the $F-4 G^{\circ}$ will be leaving. This inference states that if the source of the object of a physical tranaier is missing, then the source could be the initial location of the object. The third question can be thought of as two questions: "Which SCI (Standard Configuration Load - a predefined weapons package) are carried by an $F-4 C$ ?" and "Which of chose contain ECM (Electronic Councer Measures - radar jaming equipment)?". The first part requires a script based inference: In order for an SCL to be carried by an aircraft, the aircraft wast be capable of having the SCL as a part. After the first part is answered as a question, the second part is answered as a second question to discover which contain ECM.

The systew of representation used for nowinals (or picture producers) differs from that normally present in a CD system. Typically, an object such as an F-4C would be represented as a picture producer with a TIPE case filled by VEHICLE, a SUBTYPE case filled by aircraft, and, perhaps, a MODEL case filled by $F-4 C$. In KNOBS, the meaning representation produced by the parser is $\nabla-4 C$, the name of a frame. The set membership of this frame is indicated by links to ocher frames. F-4C is a kind of FIGHTER which is a kind of AIRPLANE which is an AIRCRAFT which is a VEHICLE which is a PICTURE PRODUCER. We feel that representing nominals in this manner allows a finer degree of discrimination than explicitly labeled cases to denote a conceptual hierarchy.

Many of the attributes of objects in the database (which are stored as value facets of slots in FRL) are represented as kinds of RELATIONS in the KNOBS system. For example, the representation of "Hahn"s Latitude" is (LATITUDE ARGUIENT (HAHN)). Noce, however, that the representation of "Hahn's aircraft" is (AIRCRAFT LOC (AT PLACE (HAHN))). PREVIOUS WORK

We would like to distinguish the KNOBS natural language facility from such familiar natural language query syscems as LADDER [Hendrix, 1978] and LUNAR [Woods, 1972] in both function and method. The functional model of the above systems is that of someone with a problem to solve and a database containing information useful in its solution which he can access via a natural language interface. KNOBS, by contrast, integrates the

natural language capability with multi-faceted problem solving support including critiquing and generating tactical plans. Our approach differs in method from these previous systems in its bottom-up, dictionary driven parsing which results in a canonical representation of the meaning of the query, its ability to perform context dependent inferences with this representation during question answering, and the use of a declarative representation of the domain to assist parsing, question answering, plan updating, and inferencing.

A syetem similar to APE-II in both its dictionary driven approach to parsing and its direct attack on word sense disambiguation is the Word Expert Parser (WEP) [Smal1, 1980]. This parser associstes a discrimination net with each word to guide the meaning selection process. Each word in a sentence is a pointer to a coroutine called a word expert which cooperates with neighboring words to build a meaning representation of the sentences in a bottom-up, i.e., data driven. fasbion. At each node in the discrimination net a multiple-choice test is executed which can query the lexical properties or expectations, (selectional restrictions (Katz, 1963)) of neighboring words, or proposed FOCUS, ACTIVITY, and DIsCOURSE modules. The sense selection process of WEP requires that each word know all of the contexts in which its senses can occur. For example, to find the meaning of "pit", the pit expert can ask if a MINING-ACTIVITY, EATING-ACTION, CAR-RACING, or MUSIC-CONCERT-ACIION is active.

APE-II evolved from APE (A Parsing Experiment), a parser used by the DSAM (Distributable Script Applying Mechaniom) and ACE (Academic Counseling Expert) projects at the University of Connecticut [Cullingford, 1982]. APE is based on the CA parser [Birnbaum, 1981] with the addition of a word sense disambiguation algorichm.

In $C A$, word definitions are represented as requesta, a type of test-action pair. The test part of a request can check lexical and semantic features of neighboring words; the actions create or connect $C D$ structures, and activate or deactivate other requests.

The method available to select the appropriate meaning of a word in $C_{A}$ is to use the test part of separate requests to examine the meanings of other words and to build a meaning representation as function of this local context. For example, if the objeet or "serve" is a food, the meaning is "bring to"; if the otject is a ball, the meaning is "hit toward". This method works well for selecting a sense of a word which has expectations. However, some words have no expectations and the intended sense is the one that is expected. For example, the proper sense of "ball" in "John kicked the ball." and "John attended the ball." is the sense which the central action expects.

The word definitions of APE are also represented as requests. A special concept called a VEL is used to represent the set of possible meanings of a word. When searching for a concept which has certain semantic features, an expectation can select one or more senses frow a VEL aud 
diacard those that are not appropriate. In addition, APE can use expectations from a contextual knovledge source such as a script applier to select a word sense. Each ocript is augmented vith parser executable expectations called named requests. For example, at a certain point in understanding a restaurant story, leaving - tip for the vaiter is expected. The parser is then given aamed requear which could help disambiguace the worda "leave" and "tip", should they appear.

$A P E-I I$

A word definition in APE-II consiste of the set of all of its senses. Each sense contains a concept, i.e., partial CD structure which expresses the meaning of this sense, and a set of conceptual and lexical expectations.

A conceptual expectation instructs the parser to look for a coacept in a certain relative ponition wich mets a selectional restriction. The expectation also contains alectional preference, a more opecific, preferred category for the expected concept (cf. (Wilks, 19721). If such a concept is found, the expectation contains information on how it can be combined with the concept which initiated the expectation. A lexical expectation instructs the pareer to look for a certain word and add a new, favored sense to it. This process is useful for predicting the function of a preposition [Reisbeck, 1976). The definition of a pronoun utilize: a context and focus wechanien to find the set of possible referents which agree with it in number and gender. THE PRONOUN IS THEN IREATED LIKE A HORD WITH MULTIPLE SENSES. The definitions of the words " $f(y$ ", "eat" and " $A / C$ " are shown in Fig. 2.

The definition of " $\mathrm{A} / \mathrm{C}$ " states that it means AIRCRAFT or AIR-CONDITIONER. APE-II uses selectional restrictions to choose the proper sense of " $A / C$ " in the question "What $A / C$ can fly from Habn?". On the other hand, in the sentence "Send 4 A/C to BE70701.", APE-II utilizes the facts that the OCA script is active, and that sending aircraft to a target is a scene of that script, to determine that " $\mathrm{A} / \mathrm{C}$ " means AIRCRAFT. In the question "What is an A/C?", APE-II uses a veaker argument to resolve the potential ambiguity. It utilizes the fact that AIRCRAFT is an object that can perform a role in the OCA scripe, while an AIR-CONDITIONER cannot.

The definition of "fly" stares that it reans FLY which is a kind of physical transfer. The expectations associated with fly state the actor of the sentence (i.e., a concept which precedes the action in a duclarative sentence, follows "by" in a passive sentence, or appears in various places in questions, etc.) is expected to be an AIRCRAFT in which case it is the OBJECT of FLY or is expected to be a BIRD in which case it is both the ACTOR and the OBJECT of the physical transfer. This is the expectation which can select the intended sense of " $\mathrm{A} / \mathrm{C}$ ". If the word "to" appears, it wight serve the function of indicating the filler of the To cace of FLY. The word "from" is given 2 similar definition, which would fill the FROM case with the object of the preposition which should be a PICTURE-PRODUCER but is preferred to be a LOCATION.

The definition of "eat" contains an expectation with selectional preference which indicates that the object is preferred to be food. This preference serves another purpose also. The object will be converted to a food if possible. Por example, if the object vere "chickea" then this conversion would assert that it is a dead and cooked chicken.

We will first discuse the parsing process an if sentences could be parsed in inolation and then explain how it is augmented to account for context. The implified parsing process conaists of adding the sences of each word to an active memory, considering the expectations, and removing concepts (senses) which are not connected to other concepts.

Hord sense disambigustion and the resolution of pronominal references are achieved by several mechanisms. Selectional restrictions can be helpful to resolve ambiguities. For example, many actions require an animate actor. If there are -everal choices for the actor, the inanipate ones will be veeded out. Conversely, if there are several choices for the main action, and the actor has been established as animate, then those actions which require an inanimate actor will be discarded. Selectional preferences sre used in addition to selectional restrictions. Por example, if "eac" has an object which is a pronoun whose poseible referents are a food and a coin, the food will be preferred and the coin discarded as a possible refereat.

A conflict resolution mechaniam is invoked if more than one concept satisfies the restrictions and preferences. This consists of using "conceptual conetraints" to determine if the CD structure which would be built is plausible. These constraints are predicates associated with $C D$ primitives. For example, the locational specifier INSIDE has a constraint which states that the contents must be smaller than the container.

The disambiguation process can make use of the knowledge structures which represent stereotypical domain information. The conflict resolution algorithm also determines if the CD structure which would be built refers to a scene in an active script and prefers to build this type of conceptualization. At the end of the parse, if there is an ambiguous nominal, the possibilities are matched against the roles of the active scripts. Nominals which can be a script role are preferred.

A planned extension to the parsing algorithm consists of augmenting the definition of a word sense with information about whether it is an uncommoniy used sense, and the contexts in which it could be used (see (Charniak, 1981)). Only some senses will be added to the active memory and if 


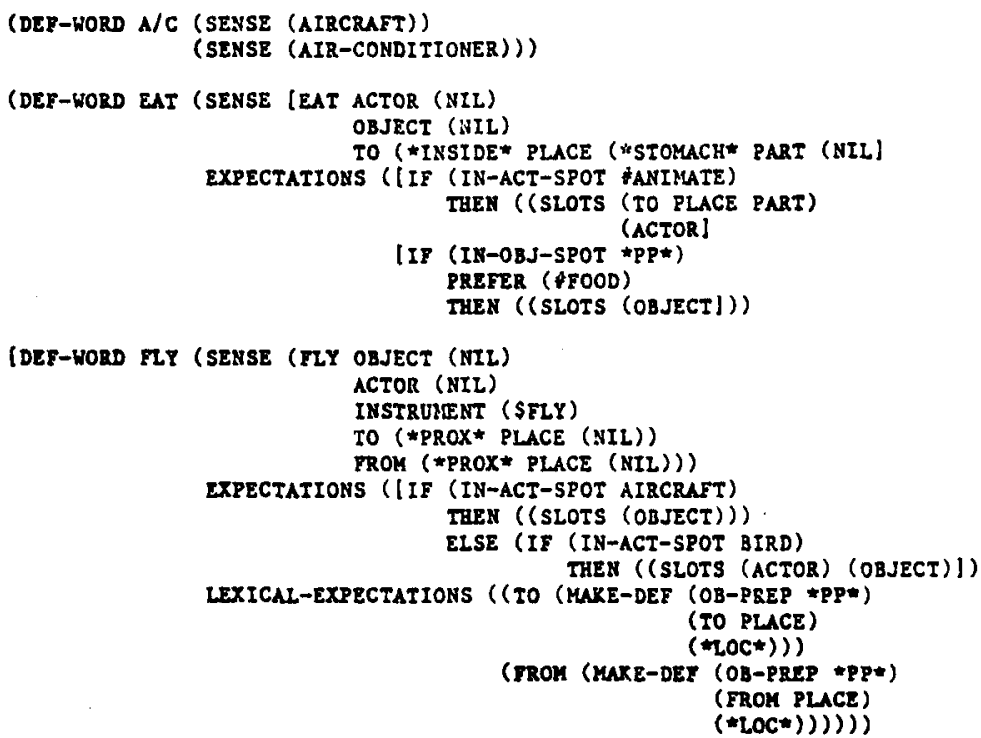

IDEF-WORD FLY (SENSE (FLY OBJECT (NIL)

ACTOR (NIL)

IXSTRURENT ( SELY)

Figure 2. APE-II Dictionary Definitions.

none of those concepts can be connected, other senses will be added. A similar mechanism can be used for potential pronoun referents, organizing concepts according to implicit or explicit focus in addition to their location in active or open focus spaces (see (Grosz, 1977)).

Another extension to APE-II will be the incorporation of a mechanism similar to the named

requests of APE. However, because the expectations of APE-II are in a declarative format, it is hoped that these requests can be generated from the causally linked scenes of the script.

\section{QUESTION ANSWERING}

After the meaning of a question has been represented, the question is answered by means of pattern-invoked rules. Typically, the patrern matching process binds variables to the major nominals in a question conceptualization. The referents of these nominals are used in executing a database query which finds the answer to the user's question. Although the question conceptualization and the answer could be used to generate a natural language response [Goldman, 1975], the current response facility merely substicutes the answer and referents in a canned response procedure associated with each question answering rule.

The question answering rules are organized according to the context in which they are appropriate, i.e., the conversational script [Lehnert, 1978], and according to the primitive of the conceptualization and the "path to the focus" of the question. The path to the focus of a question is considered to be the path of conceptual cases which leads to the subconcept in question.
A question answering production is displayed in Fig. 3. It is a default pattern designed to answer questions about which objects are at a location. This pattern is used to answer the question "What fighters do the airbases in West Germany have?". In this example, the pattern variables \&LOC is bound to the meaning representation of "the airbases in West Germany" and COBJECT is bound to the meaning representation of "fighters". The action is then executed and the referent of SOBJECT is found to be (FIGHTER) and the referent of SLOC is found to be (HAHN SEMBACH BITBURG). The fighters at each of these locations is found and the variable ANSWER is bound to the value of MAPPAIR:

((HAHN . $(F-4 C F-15))$ (SEMBACH . NIL)

(BITBURG . $(F-4 C F-15))$ ).

The response facet of the question answering production reformats the results of the action to merge locations with the same set of objects. The answer "There are none at Sembach. Hahn and Bitburg have $F-4 C s$ and $F-158 . "$ is printed on successive iterations of PMAPC.

The production in Fig. 3 is used to answer most questions about objects at a location. It invokes a general function which finds the subset of the parts of a location which belong to a certain class. The OCA (offensive counter air) script used by the KNOBS syotem contains a more specific pattern for answering question about the defenses of a location. This production is used to answer the question "What SAMs are at BE70701?". The action of this production executes a procedure which finds the subsec of the surface to air missiles whose range is greater than the distance to the location. 


\author{
(DEF-Q-PAI PAT (*EXISTS* ONJECT SOBJECT \\ LOC ("PROX* PLACE \&LOC) \\ ACTION (MAPPAIR (FIMD-REFERENTS SLOC) \\ (FUNCTION (LNABDA (LOC) \\ (MAPCONC (FIMD-RETERINTS SOBJECT) \\ (TOHCTIOA (LAMADA (TYPE). \\ (FIND-ONUECTS-AT LOC TYPE] \\ esponse (PMAPC (FERgerairs ANSWER) \\ (TOMCTION (LAMBDA (LOC ITEMS) \\ (COSD ( (MULL ITEMS) \\ (yse myere are none at " \\ (1Mits 206) \\ (n)) \\ (T (MSG (MAYR LOC)

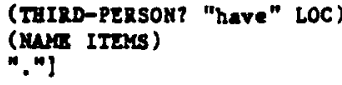$$
\text { (MNG ITEMS) }
$$ \\ Q-Pocus (ONJECT IS-A)]
}

Fiaure 3. A Ouestion Answering Production.

In addition to executing detabase query, the action of a rule can recursively invoke other question anovering rules. For example, to answer the question "How many airbases have $F-4 C^{\circ}$.?", a general rule converts the conceptualization of the question to that of "Which airbases heve F-4C'.?" and counts the rewult of anovering the latter. The question ansvering rules can eloo be used to find the referent of conplex nominals such as "the airbates which have $8-4 C^{-a "}$ ". The path to the focus of the "question" is indicated by the conceptual case of the relative pronoun.

\section{IRFERENCE}

When important roles are not filled in a concept, "conceptual completion" inferences are required to infer the fillers of conceptual cases. Our conceptual completion inferences are expresued as rules represented and organized in a manner analogous to question anowering rules. The path to the focus of a conceptual completion inference is the conceptual case which it is intended to explicate. Conceptual completion inferences are run only when neceasary, i.e., vhen required by the pattern matcher to enable a question answering pattern (or even another inference pattern) to match successfully.

An example conceptual completion inference is illustrated in Fig. 4. It is designed to infer the missing source of a physical transfer. The pattern binds the variable $\triangle O B J E C T$ to the filler of the OBJECT role and tha action executes a function which looks at the LOCATION case of SOBJECT or checks the database for the known location of the referent of $80 B J E C T$. This inference would not be used in processing the question "Whicb aircraft at

Ramstein could reach the target from Haha?" because the source has been explicitly stated. It would be used, on the other hand, in processing the question, "Which aircraft at Ramstein can resch the target?". Its effect would be to fill the FROM slot of the question conceptualization with RAMSTEIN.

\begin{abstract}
(DET-IMTERENCE PAT (\#PTRANS\# OBJECT GOBJECT) ACTION (FIND-LOCATION SOBJECT) IMTRREMCE (FROM))
\end{abstract}

Figure 4: A Concept Completion Inference.

If a question ansvering production cannot be found to respond to question, and the question refers to a ocene in an active ocript, causel inferences are used to find an answerable question which can be constructed as a state or action implied by the original question. These inferences ere repreaented by causel links [Cullingford, 1978] vhich connect the states and acrions of a stereotypical situation. The causal liaks used for this type of inferance are BrSULT (action: can result in state changes), EMABLE (ataces can enable action), and RESULT-BMAsL (an action results in a trate which enables a action). This last inference is so comon that it is given a special link. In some cases, the intermediate state is unimportant or unknown. In addition to causal links, temporal links are also represented to reason sbout the sequencing of actions.

The causel inference process consists of loceting a ecript pattern of an active script which represents the scane of the ecript referred to by a question. The pattern matching algorithm assures that the constants in the pattern are a super-class of the conetants in the conceptual hierarchy of FRL frames. The variables in script patterns are the Bcript roles which represent the common objects and actors of the script. The binding of script roles to oubconcepts of a question conceptualization is subject to the recursive matching of patterns which indicate the common features of the roles. (This will be explained in more detail in the section on interactive script instantiation.) After the scene referenced by the user question is identified, a new question concept is constructed by substituting role bindings into patterns representing states or actions linked to the identified scene.

Two script patterns from the OCA script are illuetrated in Fig. 5. The script pattern named 


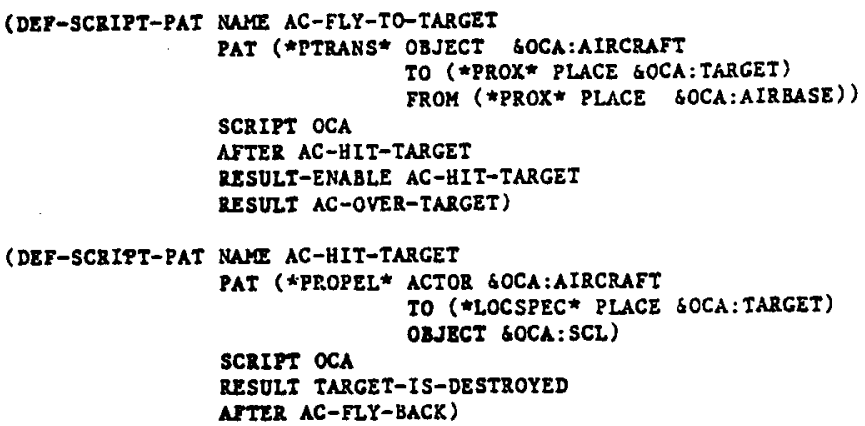

Figure 5. Definitions of Script Patterns.

AC-FLY-TO-TARGET matches the meaning of sentences which refer to the aircraft flying to the target from an airbase. It results in the aircraft being over the target which enables the aircraft to attack the target. The script pattern AC-HIT-TARGET represents the propelling of a weapon toward the target. It results in the destruction of the target, and is followed by the aircraft flying back to the airbase.

The knowledge represented by these script patterns is needed to answer the question "What aircraft at Hahn can strike BE70701?". The answer produced by KNOBS, "E-16: can reach BE70701 from Hahn.", requires a causal inference and a concept completion inference. The first step in producing this answer is to represent the meaning of the sentence. The conceptualization produced by APE-II is show in Fig. 6a. A search for a question anowering pattern to answer this fails, so causal infereace are tried. The question concept is identified to be the AC-HIT-TARGET scene of the OCA script, and the scene which RESULT-ENABLEs it, AC-ELY-TO-TARGET is instantiated. This new question conceptualization is displayed in Fig 6 b. A question answering pattern whose focus is (OBJECT IS-A) is found which could match the inferred question ( $\mathrm{Fig} .6 \mathrm{c}$ ). To enable this pattern to watch the inferred question, the FROM case must be inferred. This is accomplished by a concept completion inference which produces the complete conceptualization shown in Fig. 6d. Finally, the action and response of the question answering are executed to calculate and print an answer.

\section{INTERACTIVE SCRIPT INSTANTIATION}

The script patterns which describe the relationships among the scenes of a situation are also used by the KNOBS system to guide a conversation about that domain. The conversation with KNOBS in Fig. 7 illustrates the entering of plan components by interactively instantiating script patterns.

The first user sentence instantiates two script patterns (the flying of aircraft, and the striking of a target) and binds the script roles: TARGET to BE70501, WING to 109TEW, AIRCRAFT-NUMBER to 4, and TILE-OVER-TARGET to 0900. KNOBS asks the user to select the AIRCRAFT. Because the user replied with question whose answer is an aircraft, KNOBS ank if the user would like wou Id like to use that aircraft as a component of the developing plan. This is accomplished by a rule that in activated when KNOBS asks the user to specify a plan component. The incerpretation of the user's negative anower is handled by a rule activated when RNOBS asks a yes-no question. RNOBS checks the consistency of the user's answer and explains a constrainc which has failed. Then, the user corrects this problem, and RNOBS processes the extra information supplied by matching the meaning of the user input to a script pattern.

(\#PROPEL* TO (\#PROX* PLACE (BE70701)) ACTOR (AIRCRAFT IS-A $(* ? *)$ OBJECT (NIL) LOC (AT PLACE (HAHN))) MODE ("POTENTIAL*))

Figure 6a. The Meaning Representation of "What aircraft at Hahn can strike BE7070l?"

(*PTRANS* OBJECT (AIRCRAFT IS-A $(* ? *)$ LOC (AT PLACE (HAHA))

TO (\$PROX PLACE (BE70701) FROM (*PROX* PLACE (NIL)) - MODE (*POTENTIAL*))

Fiqure 6b. The Conceot Inferred from $6 a$. (What aircraft at Hahn can go to BE70701?)

("PTRANS* TO (\$PROX* PLACE \&TARGET) FROM (\#PROX " YLACE SAIRBASE) GBJECT KAIRCRAFT

Fiqure 6c. A Ouestion Answering Pattern which could Match $6 \mathrm{~b}$.

(*PTRANS* OBJECT (AIRCRAFT IS-A (*?*) LOC (AT PLACE (HAHN))) TO (\#PROX* PLACE (BE70701)) FROM (*PROX* PLACE (HAHN)) MODE (*POTENTIAL*) )

Fiqure 6d. The Complete Question Conceptualization after Inferring the Source. 


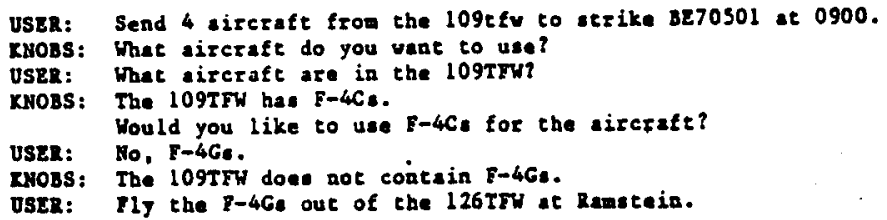

Fiaure 7. A Conversation with KNOBS.

(DET-ROLT-PAT PAT (AIRCRAFT LOC (\$PROX PLACE 6OCA:AIRBASE) PART (PART ARGUNENT SOCA:WING)) SCRIPT-ROLE SOCA:AIRCRATT SCRIPT OCA)

Figure 8. A Script Role Pattern.

A script role can be bound by matching againet patterns asociated with other script roles in addition to matching against ecript patterna. Fig. 8 showe a role pattern essociated with the script role AIRCRAPT. This patterm serves two purposes: to prevent bindinge to the script role which would not make sense ( $i . e .$, the object which plays the AIRCRAII role must be an aircraft) and to recursively bind other script roles to attached concepts. In this example, the AIRBASE or the WIRG could be attached to the AIRCRAFT concept, e.8., "Y-4C. from Babn" or "g-4C. in the 126 Trw".

The interective script interpreter is an altermative to the menu ayotem provided by KNOBS for the entering of important components of a plan to be checked for consiatency. RHOBS also provides - wens of automntically finisbing the creation of - consistent plan. This can allow an experienced mission planner to enter a plan by typing one or two sentences and hitting a key which tella KNOBS to choose the unapecified components.

\section{TRANSFERRING DOMALN}

To demonatrate their domain independence, the KNOBS System and APE-II have been provided with knowledge bases to plan and answer questions about naval "show of flag" missions. This version of KNOBS also uses FRL as a database language.

A large portion of the question answering capability uas directly applicable for a number of reasons. First of all, dictionery entries for frames are constructed automatically when they appear in a user query. The definitions of the attributes ( $\mathrm{slots}$ ) of a frame which are represented as RELATIONs are also constructed when needed. The definitions of many common words such as "be", "have", "a", "of", etc., would be useful in understanding questions in any domain. The question answering productions and concept completion inferences are separated into default and domain specific categories. Many of the simple but common queries are handled by default patterns. For example, "Which airbases have fighters?" and "What ports have cruisers?" are answered by the same defaule pattern. Currently, the Navy version of KNOBS has 3 domain specific question answering patterns, compared to 22 in the Air Force version. (There are 46 default patteras.) The toot important knowledge etructure miseing in the Navy dounin is the scripts which are needed to perform causel inferences and dielog directed planning. Therefore, the eyeter can anawer the quetion "What reapons does the Minitz have?", but can't answer "Whet veapone does the Minitz carry?".

\section{CORCLOSION}

We have argued that the processing of natural language databace queries should be driven by the mesning of the input, as determined primarily by the manings of the constituent words. The mechanisms provided for word sense celection and for the inference of miseing meaning elements utilize a variety of knowledge sources. It is believed that this approach will prove more general and extenaible than those based chiefly on the surface structure of the natural language query.

\section{ACKHOKLEDGEMENTS}

He vould like to thank Tom Fawcett, Bud Prawley, Frank Jernigan, and Ethan Scarl for their comments.

This work was supported by USAF Electronics Syoten Division under Air Force contract F19628-82-C-0001.

\section{REFERERCES}

Birnbaum, L., and Selfridge, M., "Conceptual Analyoia," in Inside Artificial Intelligence: Five Prosrame Plue Miniacures, Schank, R., Riesbeck, C. K. (eds), Lawrence Erlbaum Associates, Hillsdale, NJ, 1981 .

Charniak, E., "Six Topics in Search of a Parger: An Overviev of AI Language Research," in Proceeds of the Ith International Joint Conference on Artificial Intelligence, Vancouver, 1981 .

Cullingford, R., "Script Application: Computer Understanding of Mewspaper Stories," Research Report 116, Department of Computer Science, Yale University, 1978.

Cullingford, R. and Pazzani, M., "Word Meaning Selection in Multimodule Language-Processing Systeme," TR-82-13, EE\&CS Dept., University of Connecticut, 1982 . 
Bagelman, C., Scarl, E., and Berg, C., "Interactive Erame Instantiation," in Proc. of The First Annual Conference on Arcificial Intelligence, Stanford, 1980 .

Goldman, N., "Conceptual Generation," in Conceptual Information Processing. Schank, R. (ed), Ninth-Holland Publishing Company, 1975.

Groaz, B., "The Representation and Use of Focus in Dialog Understanding," SRI Technical Note 151, 1977 .

Hendrix, G. G., Sacerdoti, E. D., Sagalowicz, D., and Slocum, J., "Developing a Natural Language Interface to Complex Data." Associetion for. Computing Machinery Trangactions on Database Syatems, Volume 3, Number 2, June 1978.

Katz, J. S. and Fodor, J. A,, "The structure of Semantic Theory," Language, 39, 1963.

Lehnert, W., The Process of Question Ansvering $A$ Computer Simulation of Cognition, Lawrence Eribaum Associates, Inc., 1978.

Reisbeck, C., and Schank, R. C., "Comprehension by Computer: Expectation Based Analyois of Sentences in Context," Research Report \$78, Department of Computer Science Yale University, 1976.

Roberts, R. Bruce, and Goldstein, Ira P., "The FRL Manual," MII AI Lab. Memo 409, September 1977.

Schank, R., "Conceptual Dependency: A Theory of Natural Language Understanding," Cognitive

Psychologye Vol. 3, No. 4, 1972.

Sma11, S., "Word Expert Parsing: A Theory of Distributed Word-Based Natural Language Understanding," TR-954, University of Maryland 1980.

Wilks, Y., Grammar, Meaning \& The Machine Analysis of Language, London, 1972.

Woods, W. A., Kaplan, R. M., and Nash-Webber, B., "The Lunar Sciences Natural Language Information System." BBN Report 2378, Bolt, Beranek, and Newman Inc., Cambridge, MA, 1972. 